\title{
Study of the influence factors on wireless channel by setting an inside wireless environment
}

\author{
Haobo Li ${ }^{1, a}$, Haoliang $\mathrm{Li}^{2, b}$, Ruibo Chen ${ }^{3, \mathrm{c}}$ and Hailong $\mathrm{Xu}^{4, \mathrm{~d}}$ \\ Zhengzhou University, School of Information Engineering, 450001 Zhengzhou, China \\ aielihaobo@163.com, biehlli@zzu.edu.cn, c244874716@qq.com, d1452772495@qq.com
}

Key words: Wireless channel, Received signal, Power, Path loss, Antenna.

\begin{abstract}
This paper measured the influence factors on wireless channel by setting a inside wireless environment and some test models. For the same environment, the power of received signal is reduced as the increase of the distance between transmitters and receivers. The values of path loss is between 2 and 4 .In a vertical plane, at the range of $0^{\circ}$ to $180^{\circ}$ for the angle between transmitting and receiving antennas, the factors on received signal power is substantially symmetrical about $\theta=$ $90^{\circ}$. However, the left side is higher than the right because of the height of antennas and the outside environment.The effects by obstacles on received signal are different for the materials and size of obstacles. With the increases of obstacles' area, the direct signal is reduce, the diffraction signal is increase, the power of the received signal is reduced for the reduce of direct signal and increase of diffraction signal. For different species, the resistance capacity for multiply fading of OFDM is better than single-carrier signal.
\end{abstract}

\section{Introduction}

In this test, the test process can be divided into configuration signal generator, configure the transmitter, configure the receiver, the system connection, test the scene settings, test six processes. During the test, the baseband signal is generated by the signal generator and transmitted to the RF transmitter. The transmitter processes the signal and increases the signal frequency and transmits it with an omnidirectional antenna.[1] The signal is received by the receiver and analyzed by a spectrum analyzer. By changing the distance between the transmitter and the receiver, the angle of the antenna, the obstacle situation and other factors, observe the wireless channel on the received signal power, for the test results analysis.[2 3]

By setting a inside wireless environment and two test models(Fig. 1 and Fig. 2), setting the factors which may influence the received signal power.



Fig.1 double - line ground reflection

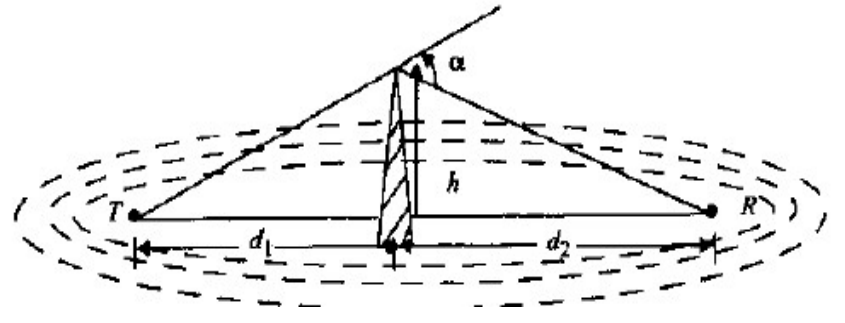

Fig. 2 Blade diffraction

\section{Test scenarios}

According to the experimental requirements, we created four test scenarios. They are used to discuss the effect of distance on the received signal, the Influence of Antenna Angle on Received Signal, the effect of the obstacle material on the received signal, and the effect of the obstacle size on the received signal. 


\section{Results and Discussions}

The effect of distance on the received signal. From the trend of the four data lines in Fig. 3, it can be seen that the power of the received signal decreases with the increase of the distance between the transmitter and the receiver (T-R) for the same signal, the greater the distance between T-R, the received signal power, the smaller. The received power can be expressed as: $P_{r}(d) d B m=\alpha-$ 10nlgd $(n=2+4 \varepsilon)(2 \leq n \leq 4)$.

We call the function polyfit and polyval of the matlab to fit the data lines, and the initial value of $d$ should set as $0.3 \mathrm{~m}$. The path loss index of the various test signals are followed by: $n_{A M}=2.8$, $n_{F M}=2.9, n_{\phi M}=2, n_{O F D M}=3.75$, conform to the theoretical value range.

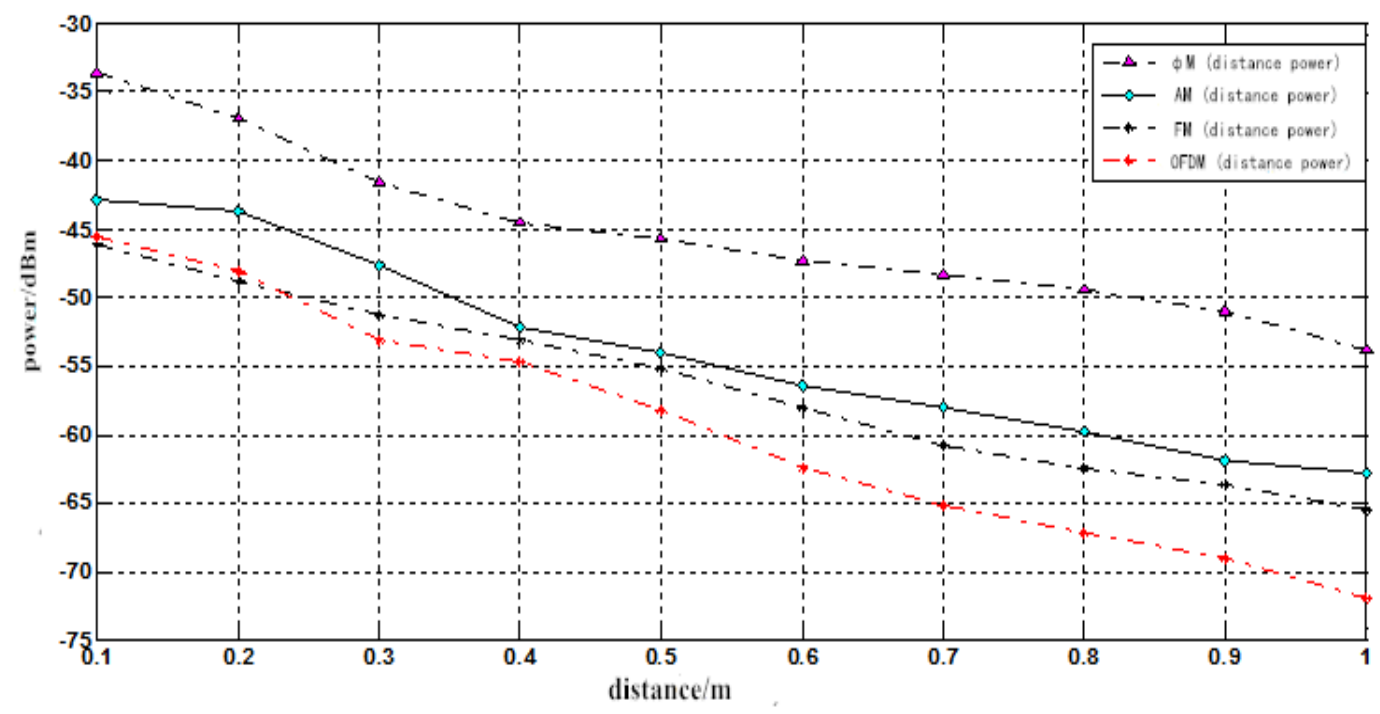

Fig. 3 The effect of distance on the received signal

The influence of Antenna Angle on Received Signal. It can be seen from the trend of the four data lines in Fig. 4 that the power of the received signal decreases with the increase of the angle of the antenna in the range of 0 to 90 degrees, and reaches the minimum value at 90 degrees. Range, the received signal power increases with the antenna angle increases, 180 degrees to reach the maximum.

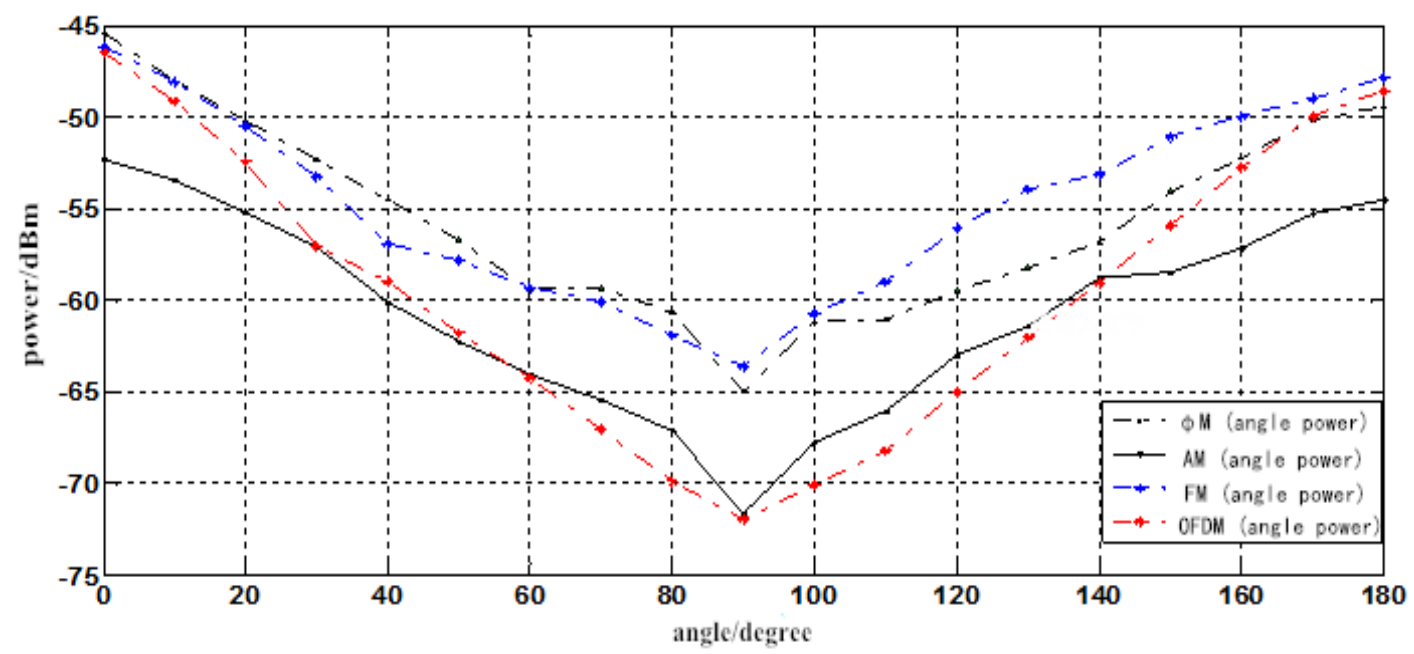

Fig. 4 Influence of Antenna Angle on Received Signal

In addition, it is observed that the change trend of the received signal power with the angle of the antenna is basically symmetrical about the angle of the antenna $=90^{\circ}$. The power value of the corresponding point on the left side of the axis of symmetry is generally greater than the right side of the axis of symmetry.

Effect of Obstacle Material on Received Signal. As can be seen from Fig. 5, when there is an 
obstacle between the T-R for the same signal, the power of the received signal becomes smaller. the material of the obstacle is different and the degree of power loss of the received signal is different. the impact of various materials on the received signal from small to large are: paper, foam, gypsum, wood. The main factor causing the change of the received signal power is the energy attenuation (transmission loss) when the wireless signal penetrates the second medium. From the three data lines in the figure, the transmission loss of the wireless signal is different for the obstacle of different materials [4 5].

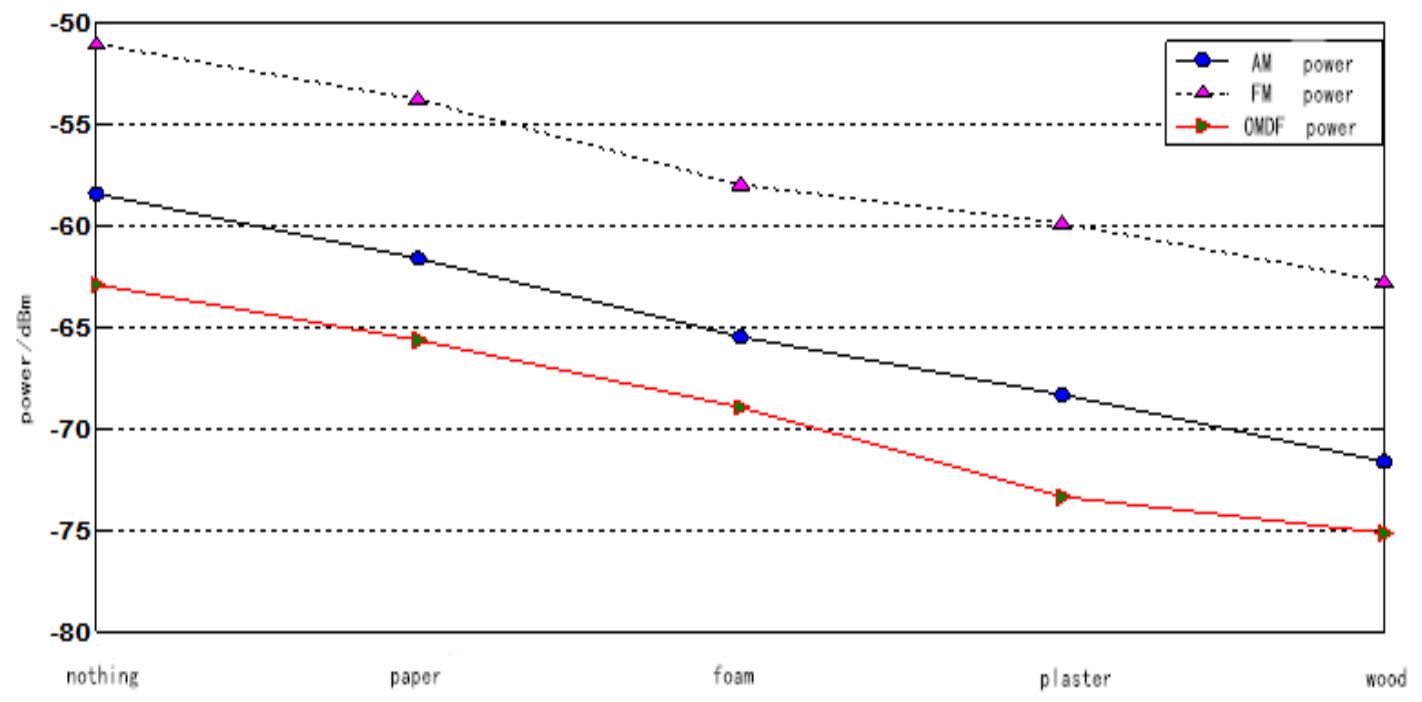

Fig. 5 Effect of Obstacle Material on Received Signal

The effect of obstacle size on the received signal. It can be seen from Fig. 6 that the influence of the received signal is increased with the increase of the length of the square obstacle in the same signal for different conditions. For the same condition, different signal and signal type are different, the obstacle to the received signal The impact is different. The data lines of the AM and FM signals are compared with the OFDM signal, and it is observed that the size of the obstacle has less influence on the OFDM reception signal than the single carrier signal [6].

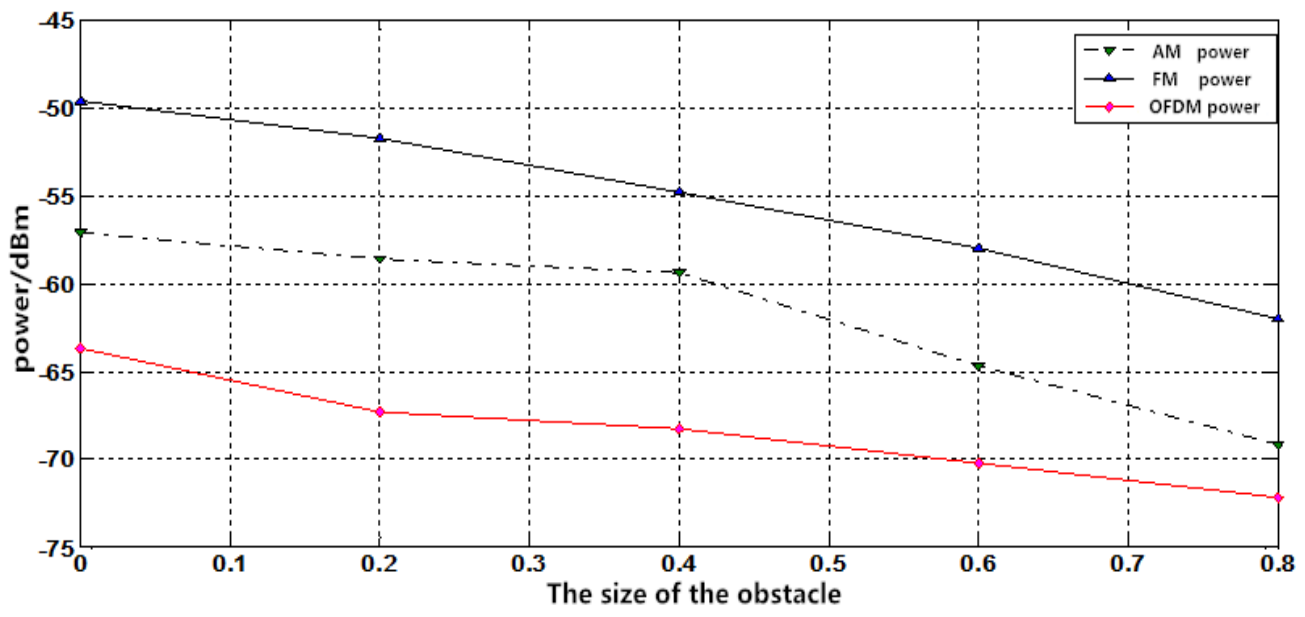

Fig. 6 The effect of obstacle size on the received signal.

\section{Conclusions}

This chapter completes the scene test for indoor wireless environments that may affect the reception of signal factor variables. Observe the test results and analyze the results: 
For the same environment, the same signal, with the transmitter and receiver distance increases, the received signal power becomes smaller, for the indoor wireless environment, the path loss factor n range of 2 to 4, the measured data consistent with the theoretical value.

For the same environment, the same signal, in the range of $0^{\circ}$ to $90^{\circ}$, with the TR antenna angle increases, the received signal power decreases. in the range of $90^{\circ}$ to $180^{\circ}$, with the TR antenna angle increases , The received signal power is increased. The power value of the received signal is substantially symmetrical about $=90^{\circ}$, and the corresponding power value on the left side of the axis of symmetry is less than the right side of the axis of symmetry.

In the same environment, the same signal, TR obstruction material is different, the power of the received signal is different, in this chapter of the test, the obstacle material on the received signal of the size of the order: wood> gypsum> foam> paper.

For the same environment, the same signal, with the TR obstacle area increases, the received signal power decreases. for the same environment, different signals, TR obstacle area changes in the size of the single carrier than the OFDM signal greater. OFDM The signal is more resistant to multipath loss.

\section{References}

[1] Hamadache Zemmour, Geneviève Baudoin, Antoine Diet: Small 'F-gain' printed slot antenna for IR-UWB wireless sensor networks applications(2014), p 8-10.

[2]Mariem Ayedi, Noura Sellami, Mohamed Siala: Throughput and energy optimization of a cooperative ARQ scheme using analog network coding, Signal Processing Conference (EUSIPCO), 2015 23rd European, p.1466-1470.

[3]Tang Liu, Hailun Xia, Chunyan Feng: A QoS-based multi-rate multicast scheme over heterogeneous cellular network, IEEE Wireless Communication Systems (ISWCS), 2016 International Symposium, p. 292-296

[4]Haiyang Zhang, Chunguo Li, Yongming Huang, Luxi Yang: Simultaneous Wireless Information and Power Transfer in a MISO Broadcast Channel with Confidential Messages”, Global Communications Conference (GLOBECOM), 2015 IEEE. p. 6-10

[5] Mrinmoy Sen, Indrajit Banerjee, Mainak Chatterjee,Tuhina Samanta: Sensor localization using received signal strength measurements for obstructed wireless sensor networks with noisy channels. 2015 IEEE Wireless Communications and Networking Conference Workshops, (WCNCW) 2015: p. 47-51.

[6] Kent Tsz Kan Cheung, Shaoshi Yang, Lajos Hanzo: Distributed Energy Spectral Efficiency Optimization for Partial/Full Interference Alignment in Multi-user Multi-relay Multi-cell MIMO Systems. IEEE Transactions on Signal Processing 2016, Vol(64): 4 p.882 - 896. 